months of 1963 the notifications for children were falling after the peak of the respiratory syncytial outbreak, while those for adults were rising as the influenza outbreak developed.

\section{Discussion}

Our findings support the view that influenza and respiratory syncytial viruses are outstandingly important as causes of epidemic respiratory disease of pneumonic severity in adults and

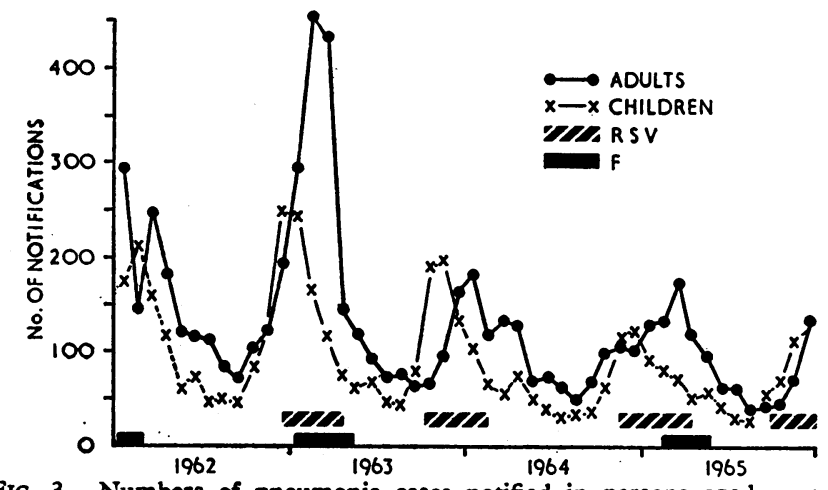

FIG. 3.-Numbers of pneumonia cases notified in persons aged over 15 and under 5 years in relation to outbreaks of influenza (F) and respiratory syncytial virus (R.S.V.) infection.

young children respectively. No other viruses have been found to cause regular elevations of such magnitude in the pneumonia statistics of the community.

In comparing the observations in adults and in young children it may be noted that postinfective immunity is usually sufficient to prevent reinfections with respiratory syncytial virus of more than trivial severity in adult life. Antigenic variation by influenza virus makes possible reinfections throughout life with periodic epidemics affecting the adult population, but it is less obvious why influenza infections in early childhood

\title{
Studies on an Epidemic of Respiratory Disease caused by Mycoplasma pneumoniae
}

\author{
T. FEIZI,* M.B., B.S., M.R.C.P. ; H. MACLEAN, $†$ M.B., CH.B., B.SC. ; R. G. SOMMERVILLE, $\ddagger$ M.D., M.C.PATH. \\ J. G. SELWYN,§ M.D., M.C.PATH.
}

Brit. med. F., 1967, 1, 457-460

Mycoplasma pneumoniae is recognized as a common cause of acute lower respiratory disease in military and civilian populations in the U.S.A. (Chanock et al., 1963 ; Forsyth et al., 1965 ; Grayston et al., 1965). Though less information is available on the prevalence of infection by that organism in this country, reports by Goodburn et al. (1963), Marmion and Hers (1963), Andrews (1965), and Watson (1965), suggest that M. pneumoniae infection is by no means rare.

We report an outbreak of acute respiratory disease in a country practice in Dumfriesshire, Scotland, which lasted from January to June 1965 , and in which $M$. pneumoniae appeared to be the commonest aetiological agent.

\footnotetext{
- Assistant Lecturer, Department of Medicinę, Royal Free Hospital, London W.C.1.

should cause so little serious illness. This paradox will be considered elsewhere.

Pneumonia and acute bronchiolitis of infants due to respiratory syncytial virus is often serious and sometimes life-threatening. The severity and periodic epidemic prevalence of this infection and its major contribution to serious respiratory disease in early life justify efforts to devise effective control measures by prophylactic vaccines or antiviral chemotherapy.

\section{Summary}

Comparison of pneumonia notifications with laboratorydiagnosed influenza and respiratory syncytial virus infections in Glasgow during 1962-5 confirmed the importance as causes of pneumonia of influenza in adults and respiratory syncytial virus in young children. Specific control measures are required for respiratory syncytial virus infections in early life.

We are grateful to Dr. W. A. Horne, Medical Officer of Health for Glasgow, for access to pneumonia statistics kindly provided by Miss Mary Knox. We also thank our clinical colleagues in hospital and general practice for their co-operation in these studies, and our technical staff for their assistance.

\section{REFERENCES}

Beem, M., Wright, F. H., Hamre, D., Egerer, R., and Oehme, M. (1960). New Engl. ₹. Med., 263, 523 .

Chanock, R. M., Kim, H. W., Vargosko, A. J., Deleva, A., Johnson, K. M., Cumming, C., and Parrott, R. H. (1961). F. Amer. med. Ass., 176, 647.

Grist, N. R. (1959). Scot. med. 7., 4, 446.

- and Landsman, J. B. (1955). Glasg. med. 7., 36, 69

Ross, C. A. C., Bell, E. J., and Stott, E. J. (1966). Diagnostic Methods in Clinical Virology. Oxford.

McClelland, L., Hilleman, M. R., Hamparian, V. V., Ketler, A., Reilly, C. M., Cornfeld, D., and Stokes, J. (1961). New Engl. F. Med., 264, 1169.

Ross, C. A. C., Stott, E. J., McMichael, S., and Crowther, I. A. (1964) Arch. ges. Virusforsch., 14, 553.

Russell, S. J. M. (1957). Scot. med. 7., 2, 88.

Stuart-Harris, C. H. (1953). Influenza and Other Virus Infections of the Respiratory Tract. London.
Of the 113 patients studied 93 were from the country practice of one of us (H.M.); the remaining 20 were patients with acute respiratory infections admitted during this period to the Royal Infirmary in Dumfries and to the near-by Lochmaben Chest Hospital. We included not only patients seeking medical attention on account of acute respiratory symptoms, but, whenever possible, other members of their families or their close contacts. Almost all the contacts questioned had had recent respiratory symptoms. In all, 21 families, in addition to 48 individual patients, were studied. Their ages ranged from 1 to 78 years.

Whenever possible blood was taken at the onset of illness and again 10 to 20 days later. Some patients sought medical attention after the first week of illness, and many of the contacts were first seen during or after the convalescent phase. Paired 
blood samples as above were taken if these patients were ill when they were first seen, and single samples were initially taken from those who had already recovered. Long-term followup extending from the 12 th to the 50th week after illness has been carried out on approximately one-third of the patients. The sera were tested by complement fixation (C.F.) with antigens of $M$. pneumoniae and Rickettsia burnetii and the following virus antigens: adenovirus, psittacosis, influenza $\mathrm{A}$, $B$, and $C$, parainfluenza 3 , respiratory syncytial, and sendai (parainfluenza 1), 2 units of complement being used; the fixation time at $4^{\circ} \mathrm{C}$. was 18 hours, and the optimum antigen dilutions were determined by chequer-board titrations against homologous hyperimmune sera.

We interpreted a fourfold or greater rise in C.F. antibody titre with paired serum samples as being diagnostic of recent infection. We included also in the recent infection group those patients with raised cold agglutinin titres and a C.F. antibody titre against $M$. pneumoniae of 64 or greater. There were many patients whose paired blood samples were not taken early in their illness or from whom we had single convalescent or paired convalescent and longterm follow-up samples. In such circumstances it was thought reasonable to classify as probable recent cases of infection those patients who had C.F. antibody titres of 64 or greater and those who on follow-up six to nine months later had a fourfold or greater fall in antibody titre. We are aware that this latter group could include some patients with residual antibodies from a previous infection and exclude those with recent infection who did not develop C.F. antibody titres of 64 . In two children aged 1 and 2 years C.F. antibody titres of 16 and 32 were also taken as evidence of probable recent infection.

In addition to the C.F. tests haemoglobin estimations, white cell and reticulocyte counts, and blood films were made; cold agglutinin titres were performed on serum separated at $37^{\circ} \mathrm{C}$., and the direct Coombs test was carried out on red cells taken into acid citrate dextrose. The Paul-Bunnell test was performed when the blood film was suggestive of glandular fever.

Chest radiography was performed only on hospital inpatients. The diagnosis in most patients was therefore based on clinical examination. A diagnosis of upper respiratory infection was made in patients with a sore throat and/or nasal symptoms, and in whom cough was absent or was a trivial symptom only. A diagnosis of acute bronchitis was made when patients had a severe cough with or without sputum and there were no clinical signs of pulmonary consolidation. A diagnosis of pneumonia was made in patients with a cough and clinical signs of consolidation. Since non-bacterial pneumonias characteristically cause radiological shadowing with relatively few clinical signs of pulmonary consolidation, it is probable that some of our patients thought to have acute bronchitis did in fact have pneumonitis. The term " myringitis" was applied to the syndrome of earache with a reddened ear-drum in the presence of a normal light reflex. Sinusitis was diagnosed when there was purulent nasal discharge associated with tenderness over the frontal or maxillary sinuses.

Though this report is concerned mainly with a detailed study of 113 patients, C.F. antibody results without haematological data on a further 92 patients with acute respiratory infections during the first six months of 1965 and on 28 such patients tested during 1964 are presented also.

\section{Results}

During the first six months of 1965 there was more than a threefold increase in the incidence of C.F. antibody against M. pneumoniae among patients with acute respiratory disease in this practice as compared with 1964 (Fig. 1). This was accompanied by a corresponding increase in the incidence of titres compatible with recent infection. Of the 113 patients studied in detail 54 had C.F. antibody at titres of $1: 8$ or greater to $M$. pneumoniae; in 40 of these the titres were compatible with

recent infection with this organism, 14 of them having titres " diagnostic" of recent infection and 26 being classed as " probable" recent cases of infection. The mode of presentation in these 40 patients is shown in Table I.

Various other infections were diagnosed in 25 patients, and in 58 a diagnosis was not established. The total number of diagnoses exceeds 113 owing to the presence of a double infection in some patients.

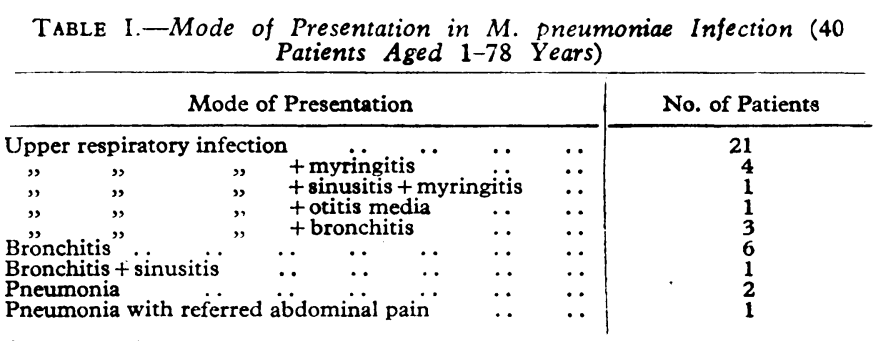

\section{Symptoms and Clinical Signs in M. pneumoniae Infection}

Tables II and III summarize the incidence of various symptoms and clinical signs among the 40 patients in the $M$. pneumoniae group. Associated infections were diagnosed in several patients, and their presence has been indicated in Tables II and III with respect to each symptom and sign. The possible role of bacterial and viral coinfection in modifying the manifestations of $M$. pneumoniae infection may be important to consider. This present study is incomplete, however, in that bacterial cultures were performed in only a minority of patients.

Cough of varying degrees of severity was the commonest symptom, occurring in 29 patients. A moderate or severe degree of malaise often necessitating confinement to bed was present in 23 patients. Often the malaise was quite out of proportion to the clinical findings; such that a normally stoic farmer would be quite unable to work for a week to 10 days with a mere upper respiratory infection. Pyrexia of $99^{\circ} \mathrm{F}$. $\left(37.2^{\circ}\right.$ C.) or above was recorded in nine patients. This is probably an underestimate of the incidence of febrile illness. If 
TaBle II.-Symptoms Associated with $M$. pneumoniae Infection in 40

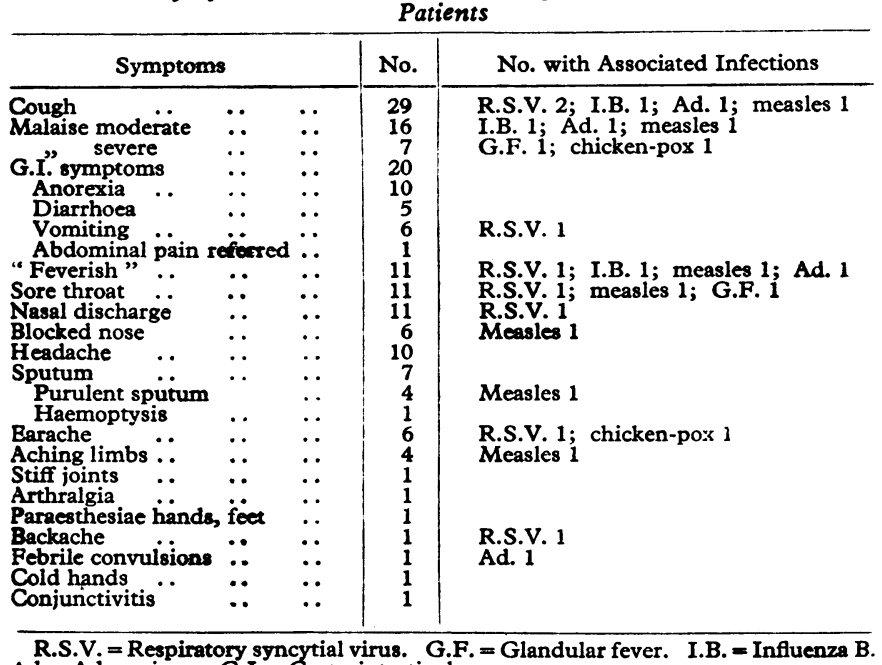

Ad. = Adenovirus. G.I. = Gastrointestinal.

TABLE III.-Clinical Signs in M. pneumoniae Infection in 40 Patients

\begin{tabular}{|c|c|c|c|}
\hline \multicolumn{2}{|l|}{ Clinical Signs } & No. & No. with Associated Infections* \\
\hline \multicolumn{2}{|c|}{ Rashes (other than " herpes ") } & 13 & Ad. 1; chicken-pox; Staph. pyogenes \\
\hline 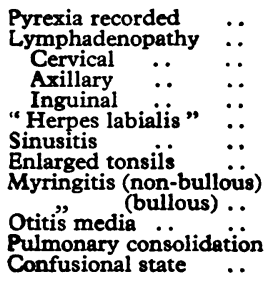 & $\begin{array}{l}\ldots \\
\cdots \\
\cdots \\
\cdots \\
\cdots \\
\because \\
\because \\
\cdots\end{array}$ & $\begin{array}{l}9 \\
7 \\
7 \\
2 \\
1 \\
6 \\
2 \\
6 \\
4 \\
1 \\
1 \\
3 \\
1\end{array}$ & $\begin{array}{l}\text { I.B. } 1 \text {; Ad. } 1 \\
\text { R.S.V. 1; G.F. 1; after chicken-pox } 1 \\
\text { R.S.V. } 1 \\
\text { G.F. } 1 \\
\text { I.B. } 1 \\
\text { Ad. 1; G.F. } 1 \\
\text { After chicken-pox 1 } \\
\text { I.B. } 1\end{array}$ \\
\hline
\end{tabular}

feverish symptoms are considered together with records of pyrexia 16 patients were either feverish or had a pyrexia recorded. Gastrointestinal symptóms were present at some time during the illness in 20 of the patients, the commonest among them being anorexia. The anorexia was often severe, and frequently lasted for two to three weeks or longer. Diarrhoea and vomiting occurred in five and six patients respectively; these were transient symptoms lasting one to three days, and occurred most commonly during the first week of illness.

Rashes and mucocutaneous lesions occurred twice as often in the $M$. pneumoniae group of patients as in patients with respiratory infections without serological evidence of $M$. pneumoniae infection. Apart from typical herpes simplex and measles, rashes occurred in 13 of the former group. Table IV lists the types of lesion observed. We should stress that it was not possible to implicate with certainty $M$. pneumoniae as a causal agent, for a great many other infections occurred.

Though direct bacteriological and virological cultures were not systematically performed, evidence of bacterial and viral coinfection in some of the patients with mucocutaneous lesions obscured the aetiology. Thus a child aged 5 years who developed a papulovesicular eruption on her face and conjunctivitis in association with $M$. pneumoniae infection yielded a coagulase-positive staphylococcus from these lesions. A 9-year-old boy developed a febrile upper respiratory infection with lymphadenopathy and a bullous myringitis while recover-

TABLE IV.-Rashes and Mucocutaneous Lesions Associated with M. pneumoniae Infection in $\mathbf{4 0}$ Patients

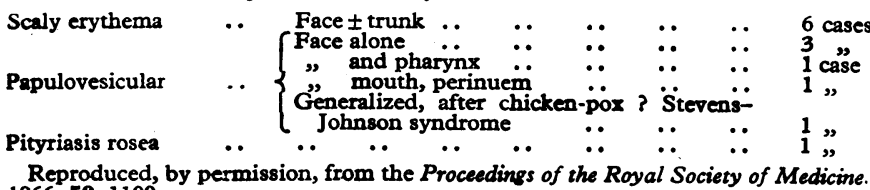
Reproduced,
1966, 59, 1109. ing from chicken-pox; he was a "probable" recent case of $M$. pneumoniae infection. This febrile episode was followed by the development of a severe vesicular eruption affecting his face, trunk, limbs, and oropharynx. This rash closely resembled Stevens-Johnson syndrome, with the exception that the conjunctivae were spared. A 3-year-old child who was admitted with a febrile upper respiratory infection and in whom a diagnosis of glandular fever and probable recent $M$. pneumoniae infection was made developed typical pityriasis rosea in the third week of illness. Rashes were not confined to patients with a complicated illness. Erythematous scaly rashes were observed in patients in whom other infections did not seem to be involved.

\section{Treatment}

The majority of patients with $M$. pneumoniae infection did not receive chemotherapy. Penicillin was the first antibiotic used in patients presenting with pneumonia and with myringitis, because bacterial pneumonia and streptococcal middle-ear disease could not be immediately excluded. If there was no clinical improvement in two to three days, or if cold agglutinin titres were found to be raised, suggesting a diagnosis of $M$. pneumoniae infection, demethylchlortetracycline (Ledermycin) was given. This drug was thus given to two patients with pneumonia and to five cases with middle-ear disease in whom a diagnosis of $M$. pneumoniae infection was subsequently confirmed. Since the drug was prescribed most often for the patients with severe disease in whom recovery was generally a gradual process, and since there were no comparable untreated patients, its effect on the duration and severity of the disease was impossible to assess.

\section{Duration of Illness}

The duration of illness was recorded in 23 patients with $M$. pneumoniae infection. In the majority of them the symptoms persisted for 7 to 10 weeks after the onset of their illness (Fig. 2). Associated infections were diagnosed in 7 out of 14 patients with such prolonged symptoms. The symptoms responsible for the prolongation of illness are shown in Table V. Malaise, cough, and recurrent respiratory symptoms were the commonest causes of prolonged illness.

TABLB V.-Symptoms Responsible for Prolonged Illness in 23 Cases of

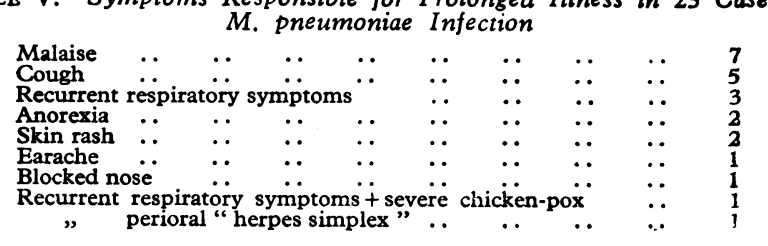

\section{Haematological Findings}

The haematological findings in this epidemic have been previously described (Feizi et al., 1966), and can be summarized as follows. Cold agglutinin titres of 64 or above (at $4^{\circ} \mathrm{C}$.) or significant changes of cold agglutinin titres were found only among patients with evidence of $M$. pneumoniae infection or their contacts. In patients who developed cold agglutinin titres of 64 , or above, the levels fell to the normal range between the 7 th and the 22nd week after illness. An interesting phenomenon of white cell agglutination was observed in two patients with high cold agglutinin titres. The white cell counts in these two patients were within the normal range. Among other patients with $M$. pneumoniae infection normal, or slightly raised, or slightly reduced total white cell counts were recorded with a variable degree of lymphocytosis. Eighty-three per cent. of patients with "undoubted" or "probable " recent infection with $M$. pneumoniae had a weakly positive direct Coombs test 
of grade + or ++ (Blood Group Reference Laboratory, London, Coombs reagent). Those with a grade ++ reaction which were tested with specific antisera gave a positive result with anticomplement (antihuman $\beta_{1}, C$ serum, Dutch Red Cross Laboratories). Though overt haemolytic anaemia did not develop, a mild reticulocytosis compatible with a low-grade haemolytic disorder commonly occurred in patients with $M$. pneumoniae infection. The incidence of a positive direct Coombs test and reticulocytosis fell with time, but in some patients they persisted as late as the 50th week after onset of illness.

\section{Discussion}

Serological tests should ideally be accompanied by attempts at direct isolation of the infecting organism in the diagnosis of $M$. pneumoniae infection. In this study the C.F. test was used as the diagnostic criterion, and it is necessary to take into account that it becomes positive in less than $80 \%$ of people infected with this organism (Chanock et al., 1963). It is none the less a convenient and useful test. A large proportion of our patients with a diagnosis of $M$. pneumoniae infection presented with upper respiratory symptoms and did not need admission to hospital. Our figures for the incidence of pneumonia are in agreement with the observations of Couch et al. (1964), who diagnosed pneumonia in only 4 out of 52 volunteers who had been experimentally inoculated with $M$. pneumoniae. In our present series only 3 out of 40 patients were diagnosed clinically as having pneumonia, and only one of these required admission to hospital. Sporadic cases of pneumonia associated with cold agglutinins and, rarely, with autoimmune haemolytic anaemia are seen at hospitals in this country from time to time, and we (T. F. and R. G. S.) have investigated some 30 such cases during the past year. Many of these have C.F. antibodies to $M$. pneumoniae compatible with recent infection. These severely ill patients probably constitute only a minority of those with $M$. pneumoniae infection, many of whom will have had infections of the upper respiratory tract or influenza-like illnesses and will not have been investigated.

The presence of viral and bacterial infections in association with $M$. pneumoniae infection is probably important to record. In fowls McMartin (1965) has shown that $M$. gallisepticum and Escherichia coli, if present together, give rise to a more severe disease than either of these two agents does alone. In this epidemic associated infections were diagnosed in half of the patients whose illness was prolonged. The possible role of recent chicken-pox infection in the development of a rash resembling Stevens-Johnson syndrome in one of our patients with probable $M$. pneumoniae infection is uncertain but worth considering.

Prolonged illness caused by an organism sensitive to tetracycline (Clyde, 1963) raises the question of therapy in $M$. pneumoniae infection. Kingston et al. (1961) in a controlled trial showed a shortening of the period of illness in patients with this infection treated with tetracycline. The diagnosis of recent $M$. pneumoniae infection by serology is not usually possible before the second or third week of illness. A raised cold agglutinin titre, which is a helpful criterion, may also take two to three weeks to develop. Direct isolation of the organism generally takes a week or longer. In the absence of a rapid and reliable diagnostic method there can be inevitable delay in the institution of specific therapy. It is therefore not surprising that the response to such delayed chemotherapy was not dramatic in our patients.

In this study high-titre cold agglutinins were encountered only among patients with undoubted recent infection with $M$. pneumoniae. Fourfold or greater changes in cold agglutinin titre were encountered among undoubted and probable recent cases of infection or their contacts. The total number of other infections diagnosed with certainty was small in this outbreak; more patients with a variety of infectious diseases need to be studied and are being studied by us with a view to ascertaining if infections other than $M$. pneumoniae can give rise to transient changes of cold agglutinin titre.

The high incidence of a positive direct Coombs test and mild reticulocytosis in $M$. pneumoniae infection was surprising, and the presence of these findings in a large number of patients with normal cold agglutinin titres was totally unexpected. The persistence of a positive direct Coombs test for longer than 18 weeks after infection (a period greater than normal red-cell lifespan) would suggest either that the causal agent is persisting after the first week of infection or that the pathological process which it initiates is persisting.

Infection by $M$. pneumoniae often gives rise to another immunological disturbance-namely, an increase in the serum immunoglobulin IgM (Feizi, 1967a). This can sometimes be very marked and not unlike the situation found in the chronic cold agglutinin syndrome with autoimmune haemolytic anaemia (Feizi, 1967b). Since the cold agglutinin associated with $M$. pneumoniae is an autoantibody, infection by this organism could well be added to the list of infective conditions associated with "autoimmune" disorders recently cited by Dameshek (1966).

\section{Summary}

During the first six months of 1965 Mycoplasma pneumoniae was a common cause of acute respiratory disease in a country practice in Dumfriesshire. Though the majority of patients presented with an upper respiratory infection, they often had a prolonged illness. The problem of early diagnosis with a view to chemotherapy is discussed.

Cold agglutinin titres of 64 and above or significant changes of cold agglutinin titre on serial testing were found only among patients with serological evidence of $M$. pneumoniae infection or their contacts.

The high incidence of a positive direct Coombs test and mild reticulocytosis were suggestive of a low-grade haemolytic disorder in the majority of patients with this infection.

We are grateful to Drs. J. Lawrie, C. Clayson, and W. A. Penman for allowing us to study patients under their care, and to Dr. D. Taylor-Robinson for his criticism. We thank Miss J. Hutchinson, Miss F. McDonald, Miss D. Langran, Mr. A. S. Bryden, Mr. M. H. G. Carson, and Mr. A. Faulds for their skilled technical help. One of us (T.F.) is in receipt of grants from the Royal Free Hospital and the Medical Research Council.

\section{REFERENCES}

Andrews, B. E. (1965). Proc. roy. Soc. Med., 58, 80.

Chanock, R. M., Mufson, M. A., Somerson, N. L., and Couch, R. B. (1963). Amer. Rev. resp. Dis., 88, Suppl. p. 218.

Couch, R. B., Cate, T. R., and Chanock, R. M. (1964). F. Amer. med. Ass., 187, 442.

Clyde, W. A. (1963). Amer. Rev. resp. Dis., 88, Suppl. p. 212.

Dameshek, W. (1966). Lancet, 1, 1268.

Feizi, T. (1967a). Proc. N.Y. Acad. Sci. In press. (1967b). Proceedings of XI International Congress of Blood Transfusion, Sydney. In press.

- Maclean, H., Sommerville, R. G., and Selwyn, J. G. (1966). Proc. roy. Soc. Med., 59, 1109.

Forsyth, B. R., Bloom, H. H., Johnson, K. M., and Chanock, R. M. (1965). f. Amer. med. Ass., 191, 364.

Goodburn, G. M., Marmion, B. P., and Kendall, E. J. C. (1963). Brit. med. F., 1, 1266.

Grayston, J. T., Alexander, E. R., Kenny, G. E., Clarke, E. R., Fremont J. C., and MacColl, W. A. (1965). F. Amer. med. Ass., 191, 369.

Kingston, J. R., Chanock, R. M., Mufson, M. A., Hellman, L. P., James, W. D., Fox, H. H., Manko, M. A., and Boyers, J. (1961). Ibid., 116, 118.

McMartin, D. A. (1965). Proc. roy. Soc. Med., 58, 79.

Marmion, B. P., and Hers, J. F. Ph. (1963). Amer. Rev. resp. Dis., 88, Suppl. p. 198.

Watson, G. I. (1965). Proc. roy. Soc. Med., 58, 84. 